\title{
Overview of the NASA TROPICS CubeSat constellation mission
}

W. J. Blackwell, S. Braun, B. Zavodsky, C. Velden, T. Greenwald, et al.

W. J. Blackwell, S. Braun, B. Zavodsky, C. Velden, T. Greenwald, D. Herndon, R. Bennartz, M. DeMaria, G. Chirokova, R. Atlas, J. Dunion, F. Marks, R. Rogers, H. Christophersen, B. Annane, "Overview of the NASA TROPICS CubeSat constellation mission," Proc. SPIE 10769, CubeSats and NanoSats for Remote Sensing II, 1076908 (18 September 2018); doi: $10.1117 / 12.2320333$

EDIE Event: SPIE Optical Engineering + Applications, 2018, San Diego, California, United States 


\title{
Overview of the NASA TROPICS CubeSat Constellation Mission
}

W. J. Blackwell*a, S. Braun ${ }^{\mathrm{b}}$, B. Zavodsky ${ }^{\mathrm{c}}$, C. Velden ${ }^{\mathrm{d}}$, T. Greenwald ${ }^{\mathrm{d}}$, D. Herndon ${ }^{\mathrm{d}}$, R. Bennartz ${ }^{\mathrm{e}}$,

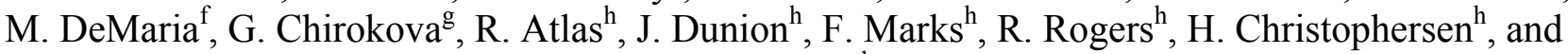
B. Annane ${ }^{\mathrm{h}}$

${ }^{a}$ Lincoln Laboratory, Massachusetts Insititute of Technology, 244 Wood Street, Lexington, MA, USA 02421-6426; ' National Aeronautics and Space Administration (NASA) Goddard Space Flight Center, 8800 Greenbelt Rd, Greenbelt, MD, USA, 20771; 'NASA Marshall Space Flight Center,

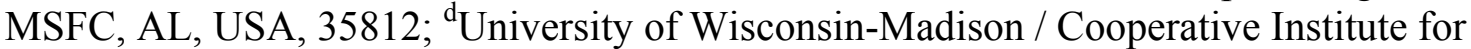
Meteorological Satellite Studies, 1225 W. Dayton St. Madison, WI, USA, 53706; 'Vanderbilt University, 2201 West End Ave, Nashville, TN, USA, 37235; ${ }^{\mathrm{f}}$ National Oceanic and Atmospheric Administration (NOAA) National Hurricane Center, 11691 SW 17th Street, Miami, FL, USA, 33165; ${ }^{g}$ Colorado State University / Cooperative Institute for Research in the Atmosphere, 3925A West Laporte Ave., Fort Collins, CO, USA, 80523-1375; ${ }^{h}$ NOAA / Atlantic Oceanographic \& Meteorological Laboratory / Hurricane Research Division, 4301 Rickenbacker Causeway, Miami, FL, USA, 33149

\begin{abstract}
Recent technology advances in miniature microwave radiometers that can be hosted on very small satellites has made possible a new class of affordable constellation missions that provide very high revisit rates of tropical cyclones and other severe weather. The Time-Resolved Observations of Precipitation structure and storm Intensity with a Constellation of Smallsats (TROPICS) mission was selected by NASA as part of the Earth Venture-Instrument (EVI-3) program and is now in development with planned launch readiness in late 2019. The overarching goal for TROPICS is to provide nearly all-weather observations of 3-D temperature and humidity, as well as cloud ice and precipitation horizontal structure, at high temporal resolution to conduct high-value science investigations of tropical cyclones (TCs). TROPICS will provide rapid-refresh microwave measurements (median refresh rate better than 60 minutes for the baseline mission) over the tropics that can be used to observe the thermodynamics of the troposphere and precipitation structure for storm systems at the mesoscale and synoptic scale over the entire storm lifecycle. TROPICS will comprise a constellation of at least six CubeSats in three low-Earth orbital planes. Each CubeSat will host a high performance radiometer to provide temperature profiles using seven channels near the $118.75 \mathrm{GHz}$ oxygen absorption line, water vapor profiles using three channels near the $183 \mathrm{GHz}$ water vapor absorption line, imagery in a single channel near 90 $\mathrm{GHz}$ for precipitation measurements (when combined with higher resolution water vapor channels), and a single channel at $205 \mathrm{GHz}$ that is more sensitive to precipitation-sized ice particles and low-level moisture. This observing system offers an unprecedented combination of horizontal and temporal resolution in the microwave spectrum to measure environmental and inner-core conditions for TCs on a nearly global scale and is a major leap forward in the temporal resolution of several key parameters needed for assimilation into advanced data assimilation systems capable of utilizing rapid-update radiance or retrieval data. Here, we provide an overview of the mission and an update on current status, with a focus on unique characteristics of the Cubesat system, recent performance simulations on a range of observables to be provided by the constellation, and a summary of science applications.
\end{abstract}

Keywords: cubesat, smallsat, remote sensing, passive microwave, temperature and moisture soundings, precipitation, tropical cyclones

\section{INTRODUCTION}

Nearly 60 million Americans live in counties that border the Atlantic and Gulf of Mexico-areas that are prone to impacts from tropical cyclones (TCs). The advent of satellite data assimilation has resulted in improved forecasts of storm tracks; however, smaller improvements have been realized in numerical forecasts of storm intensity and size. Additionally, for situational awareness of hurricane conditions, forecasters use visible and infrared imagery from 
geostationary satellites, such as from the National Oceanic and Atmospheric Administration (NOAA) Geostationary Operational Environmental Series (GOES). Advances in technology_realized through the Advanced Baseline Imager $(\mathrm{ABI})^{1,2}$ - now allow for rapid scanning of storms every 60 seconds; however, ABI is limited in spectral resolution to visible and infrared channels, which mostly allow for analysis of cloud tops in tropical systems. The international community flies a constellation of passive microwave (PMW) imagers and sounders, which can spectrally "see through" clouds to analyze temperature, moisture, and precipitation features that relate to storm dynamics. However, even with the constellation of satellites, the temporal resolution of the data is nominally 3 hours, which does not allow for the diagnosis of rapidly-evolving features critical for answering long-standing science questions related to the dynamics of rapid TC intensification.

To respond to this current gap in fine temporal-scale PMV satellite observations, the Time-Resolved Observations of Precipitation structure and storm Intensity with a Constellation of Smallsats (TROPICS) mission ${ }^{3}$ will implement a space-borne earth observation system designed to collect measurements over the tropical latitudes to observe the thermodynamics and precipitation structures of TCs over much of the storm systems' lifecycles. TROPICS is comprised of a constellation of small-scale spectrometers, developed at the Massachusetts Institute of Technology (MIT) Lincoln Laboratory (LL). TROPICS is funded through the NASA Earth Venture Instrument (EVI-3) program. The measurements will provide nearly all-weather observations of three-dimension (3D) temperature and humidity, as well as cloud ice, precipitation horizontal structure, and instantaneous surface rain rates. These measurements and the increased temporal resolution provided by the CubeSat constellation are needed to better understand TC lifecycles and the environmental factors that affect the intensification of TCs. The TROPICS CubeSats will be launched on NASAprovided expendable launch vehicles as either the primary or secondary payload to form a multi-plane constellation capable of providing median observation revisit rates (around 60 minutes), which is necessary to fulfill all threshold science requirements.

The TROPICS core instrument is a cross-track scanning PMW spectrometer that provides measurements of upwelling thermal emission and scattering of Earth's atmosphere. Measurements are taken in 12 channels near atmospheric absorption features due to oxygen and water vapor. Processing of the raw radiance values measured by the spectrometer yields atmospheric temperature, moisture, rain rates, and other information relevant to precipitation structure and storm intensity. Instrumentation needed to make these measurements has been used in space for decades, and ultra-compact instrumentation for CubeSat implementation is now available with high technology readiness level.

The scientific goal of TROPICS is to provide nearly all-weather observations of 3D temperature and humidity, as well as cloud ice and precipitation horizontal structure, at high temporal resolution-compared to current PMW measurements - to conduct high-value science investigations of TCs. More details related to the observatory can be found in Section 2; more details related to the science questions covered by TROPICS can be found in Section 3.

\section{THE TROPICS OBSERVATORY}

TROPICS comprises a constellation of six identical Space Vehicles (SVs) conforming to the $3 \mathrm{U}$ CubeSat form factor and hosting a passive microwave spectrometer payload. The constellation members will be flown in a circular Low Earth Orbit (LEO) in nearly equally-spaced orbital planes, with multiple satellites populating each orbital plane. Each orbit inclination will be roughly $30^{\circ}$. The constellation will allow for rapid-revisit sampling of vertical temperature and moisture profiles of TCs. Figure 1 shows the median revisit, the mean revisit, and the fraction of time measurements are observed with revisit rates greater than two hours as a function of the constellation orbital configuration. Three orbital planes are considered, with some number of satellites populating each orbital plane (up to four in each plane). The satellites are ejected upon deployment with unique velocities to virtually eliminate the possibility of multi-plane satellite conjunctions (satellites overlapping simultaneously in multiple orbital planes), even when the satellites are allowed to drift freely with no active control ("random phasing"). The revisit rate of the constellation is a function of latitude, and the historical frequency of named tropical cyclone is also a function of latitude. To create a scalar revisit metric, the constellation revisit rate as a function of latitude is weighted by the historical storm frequency as a function of latitude. The TROPICS baseline mission will fly six satellites (two in each of three planes), which will allow the baseline mission requirement of a 60-minute median revisit to be achieved even with the failure of up to two satellites. Also shown in Fig. 1 is a cumulative distribution function of the revisit rates. 


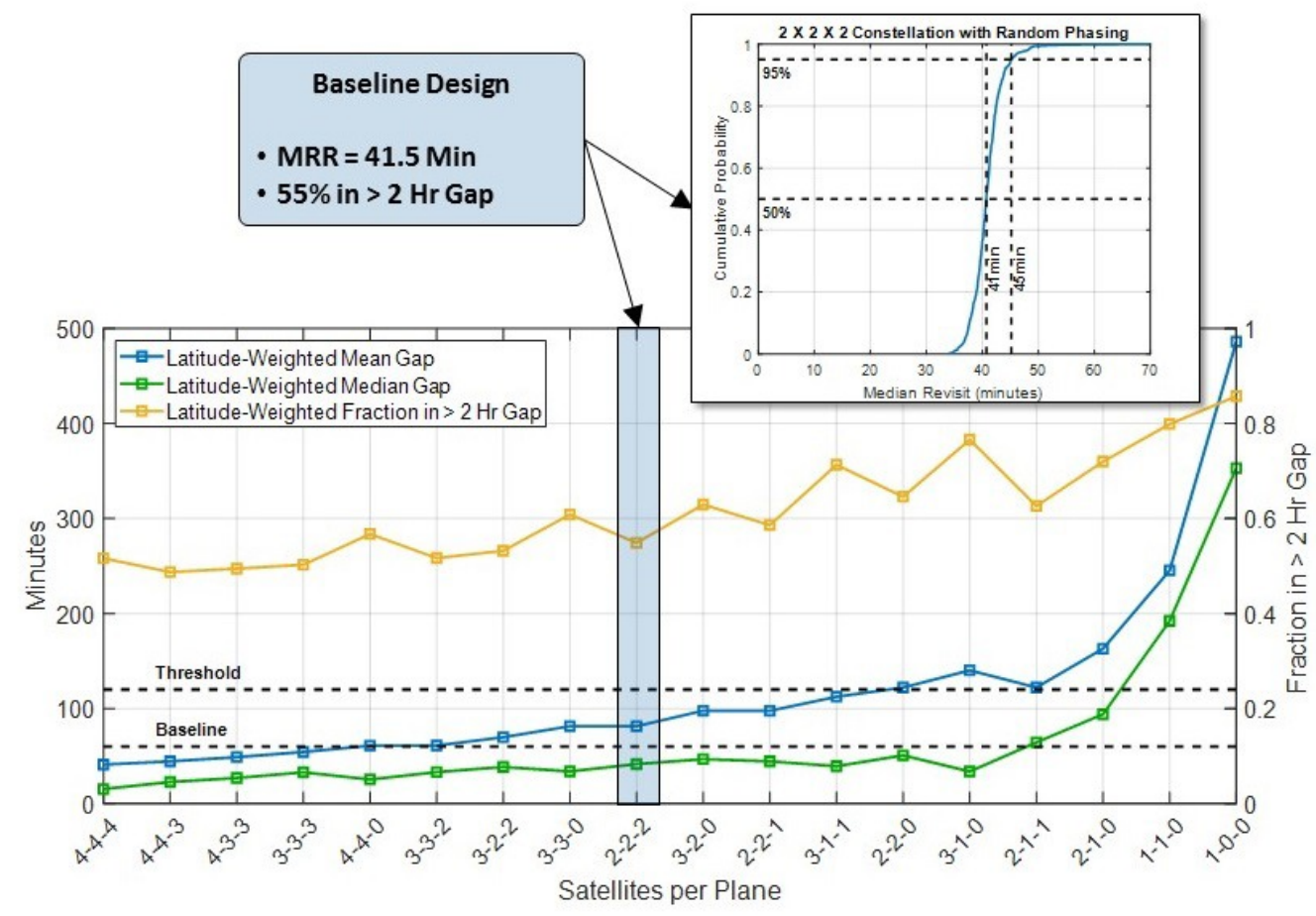

Figure 1. TROPICS constellation revisit statistics. The baseline mission case is shown shaded in blue. Other configurations are shown for comparison. The baseline requirement for median revisit of 60 minutes is indicated with a black horizontal dashed line, as is the

120 minute threshold requirement. The upper panel shows a cumulative distribution function of revisit rates of TROPICS.

Table 1. TROPICS Data Products

\begin{tabular}{|l|l|l|}
\hline Designation & Description & Requirement \\
\hline Level 0 & $\begin{array}{l}\text { Raw CCSDS payload and telemetry from } \\
\text { SVs }\end{array}$ & N/A \\
\hline Level 1a & $\begin{array}{l}\text { Timestamped, geolocated, calibrated antenna } \\
\text { temperature }\end{array}$ & See Table 2 \\
\hline Level 1b & $\begin{array}{l}\text { Timestamped, geolocated, calibrated antenna } \\
\text { temperature with bias removed }\end{array}$ & See Table 2 \\
\hline Level 2a & $\begin{array}{l}\text { Spatially resampled G-band brightness } \\
\text { temperature to F-band resolution }\end{array}$ & N/A \\
\hline Level 2b & $\begin{array}{l}\text { Atmospheric Vertical Temperature Profile } \\
(\mathrm{K})\end{array}$ & $2 \mathrm{~K}$ RMS at 50-km scan-averaged spatial resolution \\
\hline Level 2b & $\begin{array}{l}\text { Atmospheric Vertical Moisture Profile (g/g) } \\
\text { Level 2b }\end{array}$ & 25\% at 25-km scan-averaged spatial resolution \\
\hline Level 2b & $\begin{array}{l}\text { TC Intensity: Minimum Sea Level Pressure } \\
(\mathrm{hPa})\end{array}$ & $10 \mathrm{hPa}$ RMS \\
\hline Level 2b & $\begin{array}{l}\text { TC Intensity: Maximum Sustained Wind } \\
(\mathrm{m} / \mathrm{s})\end{array}$ & $6 \mathrm{~m} / \mathrm{s}$ RMS \\
\hline
\end{tabular}


The PMW spectrometer antenna is mounted on a rotating axis that will spin about the long axis of the SV. The long axis is aligned to the satellite velocity vector such that the spectrometer will record measurements along a line perpendicular to the satellite velocity in a "cross-track-scan" fashion that maximizes the area scan rate of the instrument. Each SV will record the raw passive microwave data and relay the raw data to the ground, where the data will be processed to produce the temperature and moisture profiles. Spatial resolution (averaged over the swath) is approximately $25 \mathrm{~km}$ for the moisture channels $(183-206 \mathrm{GHz})$ and approximately $40 \mathrm{~km}$ for the temperature channels $(90-118 \mathrm{GHz})$. More details on resolution can be found in Section 2.2. Radiometric data are calibrated using an onboard noise diode reference that is turned on and off against the cold sky background at least once per revolution (every two seconds).

The TROPICS observatory will provide a set of products as shown in Table 1, including raw (uncalibrated) radiances, calibrated and geolocated radiances, temperature and moisture profiles, rain rate, and intensity indicators. Details on these products and their use in the TROPICS science program is described below.

\subsection{Space Vehicles}

Each SV in the 6-member TROPICS constellation is an identical 3U CubeSat consisting of a MIT LL-built spectrometer payload integrated onto a commercially-procured bus. The spectrometer payload consists of a rotating passive Radio Frequency (RF) antenna measuring spectral radiance as it rotates about the SV velocity vector. The spectrometer uses heritage technology flown on the MicroMAS-2a CubeSat ${ }^{4}$-a $3 \mathrm{U}$ scanning CubeSat with a dual spinner payload that was launched on January 11th, 2018. MicroMAS-2b is scheduled for launch in Fall 2018. MicroMAS-2a provided the first ever CubeSat microwave radiometer cross-track sounding data (NASA GSFC IceCube provided imagery in a single channel near $883 \mathrm{GHz}$ ). The engineering team has modified the design of the MicroMAS-2 in order to meet TROPICS performance and mission reliability requirements.

The redesign includes:

- antenna modification to optimize ground profile while minimizing side lobes

- noise reduction in analog front end

- higher-dynamic-range analog-to-digital converter

- modifications to spectrometer channel center frequencies and bandwidths

- higher-reliability control electronics

- higher-reliability and lower-power motor-scanner assembly

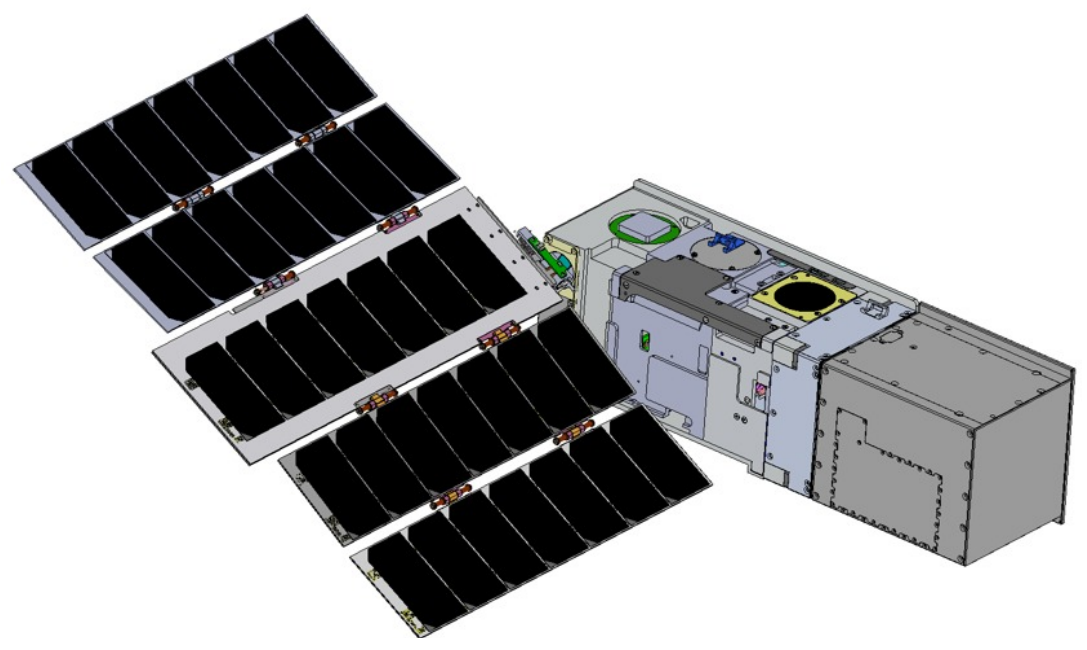

Figure 2. TROPICS Space Vehicle showing CubeSat bus, radiometer payload, and deployed articulated solar array. 
The redesign effort does not include any high-risk modifications and should simplify the build and calibration of the payload relative to the MicroMAS-2 baseline design. A notional SV including the bus and payload is shown in Figure 2. The MicroMAS-2 bus does not have sufficient pointing accuracy or power generation capability to meet TROPICS mission requirements. The TROPICS bus will match much of the functionality of the MicroMAS-2 bus, but will take advantage of recent commercial advances in CubeSat reliability and bus technology. In particular, making use of available GPS receivers for position knowledge and star cameras for attitude knowledge will greatly enhance the data product geolocation accuracy.

The spacecraft bus is being procured from Blue Canyon Technologies (BCT) and will provide power \& power conditioning, communications, on-board processing, thermal management, and attitude determination \& control system to the satellite. The flight software will provide command and control of the payload, and will interface with the bus communications system to manage payload commands and prepare payload telemetry for downlink.

\subsection{Radiometer Characteristics}

TROPICS will continue a long history of microwave sounding/imaging missions targeted to the study of storms. For example, the Global Precipitation Measurement Mission (GPM) ${ }^{5}$ and the Megha-Tropiques Mission ${ }^{6}$ both observe storms and severe weather with very high measurement fidelity but very low temporal resolution. TROPICS will fly two total power radiometers that measure 12 channels spanning approximately 90 to $206 \mathrm{GHz}$. The "WF-band" radiometer comprises eight channels from 90-119 GHz, and the "G-band" radiometer comprises four channels from 183-206 GHz. The specific channel properties are shown in Table 2. The full-width at half maximum antenna beamwidths are achieved using an offset parabolic reflector illuminated with two feed horns that are physically separated, and the beams are combined and collocated using a polarizing wire grid diplexer. Beam efficiencies for the temperature and water vapor sounding channels are designed to exceed $95 \%$. Radiometer calibration is accomplished using weakly coupled noise diodes with known and stable noise output that are turned on and off against the cold space background. Satellite intercalibration is optimized using cross comparisons ${ }^{7}$ and daily 179 calculated numerical model residuals ${ }^{8}$ to derive and implement any needed bias corrections.

Table 2. Description of the TROPICS Radiometer Channels

\begin{tabular}{|c|c|c|c|c|c|}
\hline Chan. & $\begin{array}{c}\text { Center Freq. } \\
\text { (GHz) }\end{array}$ & $\begin{array}{c}\text { Bandwidth } \\
\text { (MHz) }\end{array}$ & $\begin{array}{c}\text { Beamwidth (deg.) } \\
\text { Down/Cross }\end{array}$ & $\Delta \mathbf{T}_{\text {rms }}$ & $\begin{array}{c}\text { Cal. Acc. } \\
\text { (K) }\end{array}$ \\
\hline 1 & $91.655 \pm 1.4$ & 1000 & $2.8 / 2.98$ & 0.6 & 2.0 \\
\hline 2 & 114.50 & 1000 & $2.3 / 2.52$ & 0.75 & 1.5 \\
\hline 3 & 115.95 & 800 & $2.3 / 2.52$ & 0.85 & 1.5 \\
\hline 4 & 116.65 & 600 & $2.3 / 2.52$ & 0.8 & 1.5 \\
\hline 5 & 117.25 & 600 & $2.3 / 2.62$ & 0.75 & 1.5 \\
\hline 6 & 117.80 & 500 & $2.3 / 2.62$ & 0.8 & 1.5 \\
\hline 7 & 118.24 & 380 & $2.3 / 2.62$ & 0.9 & 1.5 \\
\hline 8 & 118.58 & 300 & $2.3 / 2.62$ & 1.0 & 1.5 \\
\hline 9 & 184.41 & 2000 & $1.5 / 1.87$ & 0.9 & 1.0 \\
\hline 10 & 186.51 & 2000 & $1.5 / 1.87$ & 0.9 & 1.0 \\
\hline 11 & 190.31 & 2000 & $1.5 / 1.87$ & 0.9 & 1.0 \\
\hline 12 & 204.8 & 2000 & $1.4 / 1.83$ & 0.9 & 1.0 \\
\hline
\end{tabular}

The radiometer operates in an "integrate-while-scanning" mode that results in elongated footprints in the cross-track direction. The spatial resolution is thus reported as the geometric mean of the minor and major axes of the ellipse projected on the earth, also accounting for earth curvature. As the constellation of six satellites scans the earth, the footprints near the edge of the scan are revisited more often than the footprints near nadir. This effect is quantified by calculating an "effective" spatial resolution that weights the spatial resolution of each footprint by the relative frequency by which it is revisited. The nadir, mean-across-scan, and effective spatial resolutions are shown in Table 3 . The satellite 
pointing accuracy and sensor mounting requirements are set to ensure geolocation errors are smaller than approximately $10 \%$ of the footprint size.

Temperature weighting functions for all 12 TROPICS channels are shown in Fig. 3. Channel passbands are designed to span altitudes from the surface up to $20 \mathrm{~km}$ for temperature and 10-km for water vapor. Multiple temperature channels probe the upper troposphere to observe tropical cyclone warm core anomalies.

(a)

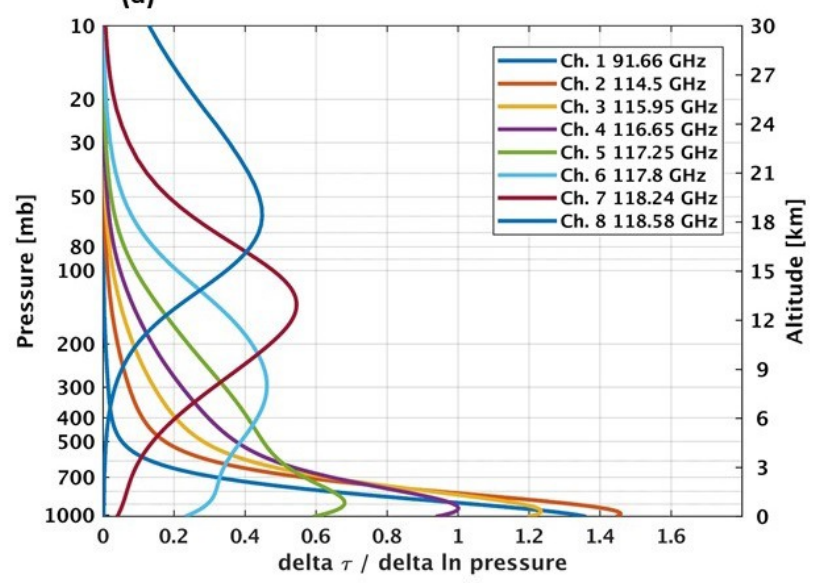

(b)

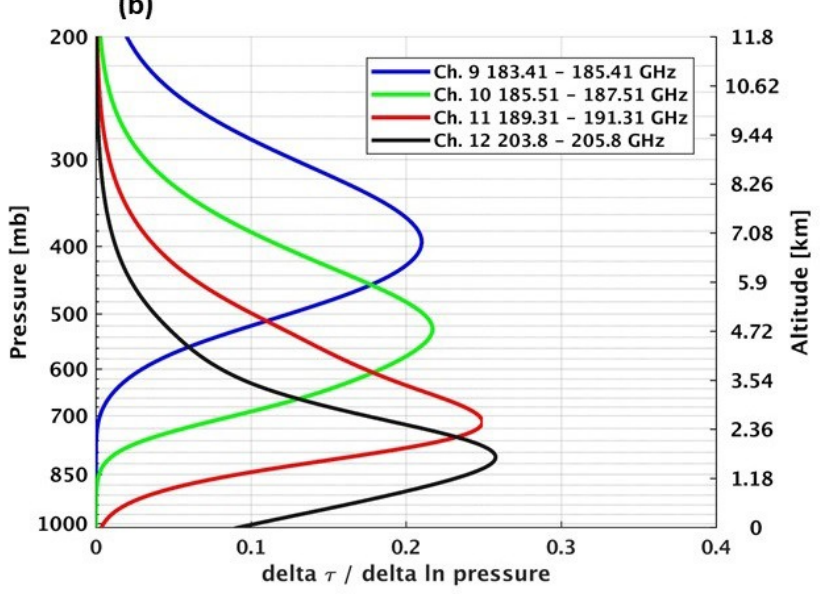

Figure 3. Weighting functions calculated at nadir incidence over a perfectly emissive surface for a standard tropical atmosphere for both a) temperature/imaging and b) water vapor/imaging channels.

Table 3. TROPICS spatial resolution for different channel subsets averaged over the 81 footprints in the swath. Effective spatial resolution refers to how often the footprints are revisited across the scan (see text for details).

\begin{tabular}{|c|c|c|c|}
\hline Band & Nadir (km) & Scan Mean (km) & Effective Across Scan (km) \\
\hline W $(90 \mathrm{GHz})$ & 27.7 & 42.9 & 50.7 \\
\hline F $(118 \mathrm{GHz})$ & 23.1 & 34.9 & 41.2 \\
\hline G $(183 \mathrm{GHz})$ & 16.1 & 23.3 & 27.5 \\
\hline G $(205 \mathrm{GHz})$ & 15.6 & 22.1 & 26.0 \\
\hline
\end{tabular}

\subsection{Ground Station and Data Processing}

The TROPICS SVs will interface with the KSAT-lite ground station network to allow for satellite command and control and downlink of bus and payload telemetry for each CubeSat in the constellation. Mission operations will be provided by BCT. MIT LL will interact with BCT to acquire the down-linked raw science data and format it into data products that can be shared with the data processing center at the University of Wisconsin (UW) Space Science \& Engineering Center (SSEC). Stored mission data will be downlinked at S-band. The data products will be made available to the data processing center via a secured connection. The data will be stored at MIT LL in a SQL database on a MIT LL computer system that includes disk redundancy and data backups. The entire mission data set will be stored at the NASA Goddard Earth Sciences Data and Information Services Center (GES DISC) enabling access by the entire scientific community. The mission data will be available in NetCDF format per discussion with NASA Earth Science Data Systems (ESDS). Other formats may be supported outside of the mission to enable improved utilization by other user communities outside of research organizations. TROPICS data have a latency requirement of 4 days but is expected to exceed that to be (on average) 24 hours in the current data downlink configuration (single downlink site) due to the costs associated with the data delivery and the cost-capped nature of the mission.

\section{SCIENCE OBJECTIVES AND APPLICATIONS}

\subsection{TROPICS Science Objectives}

The fundamental physical parameters required to address the TROPICS science objectives are 3D atmospheric temperature and humidity, storm intensity, and horizontal precipitation structure. These parameters have a long heritage 
of being derived from space-borne PMW imagery and sounding channels. Practical considerations of antenna and instrument size and mass for a CubeSat system guide the selection of PMW channels for TROPICS. Temperature and moisture profiles are retrievable from seven channels near $118 \mathrm{GHz}$ and three near $183 \mathrm{GHz}$, respectively. Precipitation structure is obtained from a combination of $90 \mathrm{GHz}, 206 \mathrm{GHz}$, and the temperature and moisture channels, with horizontal resolution matching that of the moisture data due to the high sensitivity to precipitation hydrometeors at 183 GHz. The 206-GHz channel will be sensitive to smaller ice particles than the 90-GHz channel and will generally produce a stronger signal.

Temperature sounding performance of $2 \mathrm{~K}$ RMS up to $50 \mathrm{hPa}$ (approximately $20 \mathrm{~km}$ altitude) provided by TROPICS allows sensing of upper tropospheric TC warm cores, important since a fully resolvable TC warm core is desired for objective estimates of storm intensity. The ATMS temperature sounding requirement drives the TROPICS sensor sensitivity requirement to approximately $0.5 \mathrm{~K}$ at the native sensor horizontal resolution, as determined using simulations of temperature profile retrieval performance with the TROPICS bands. Techniques developed to estimate the intensity of TCs from microwave sounder information have greatly aided TC satellite analysts and warning centers around the globe.

These techniques measure the upper-level warm-core anomaly and relate it to TC intensity assuming hydrostatic principles and statistical relationships. The upper-level thermal anomalies associated with the TC warm core are computed from brightness temperature (TB) fields for selected microwave channels. To compute the local anomaly, a core radiance value is taken from the warmest pixel near the TC center. Environmental values are selected from a filtered pattern surrounding the TC and averaged. The resulting brightness temperature anomalies are then correlated with coincident in-situ aircraft intensity data to develop regression equations.

High-frequency PMW observations provide a wealth of information on scattering by precipitation-sized ice particles. The novel $206 \mathrm{GHz}$ channel will be particularly sensitive to ice particle scattering and will provide an opportunity to better identify and map convective precipitation. All TROPICS channels together provide some information on vertical structure and will allow the derivation of proxies for intensity of precipitation in TCs. These methodologies will be modified to the combination of $90,118,183$, and $206 \mathrm{GHz}$ to arrive at brightness temperature depressions and differential scattering parameters between different channels with different gas absorption strength. Scattering signatures will be tied back to hydrometeor content and height of the scattering layer using a set of high-resolution simulations of tropical storms derived from the state-of-the-art 3D modeling system. More details on how the mission will respond to specific science questions can be found in Blackwell et al. (2018) ${ }^{3}$.

As such, the science objectives for TROPICS include:

1. Relationships between rapidly evolving precipitation structure, upper-level warm-core evolution, and associated storm intensity changes;

2. The evolution of TC precipitation structure and storm intensification in relation to environmental humidity fields; and

3. The impact of TROPICS rapid-update microwave observations on numerical and statistical intensity forecasts of TCs.

While the core mission objectives focus on TC applications, the proposed constellation can certainly address a much wider range of science goals including analysis of tropical mesoscale convective systems (MCSs), the Madden-Julian Oscillation (MJO), and monsoon weather. The data themselves also have potential to be usable for other hydrologicrelated applications, such as flood and drought monitoring and forecasting.

\subsection{TROPICS Proxy Data and Applications}

In order to better understand the characteristics of the expected mission data and to begin engagement with the applications community, proxy datasets are being developed. The primary proxy dataset is generated using an 11-day Hurricane Nature Run (HNR) ${ }^{9}$, which simulates a single low category four storm in the Atlantic basin using the Weather Research and Forecasting (WRF) ${ }^{10}$ numerical weather prediction model. Simulated data are derived using the Community Radiative Transfer Model (CRTM) ${ }^{11}$. Cross-track spatial sampling is used for an idealized antenna pattern and a boxcar channel set is used for idealized spectral response. Orbital parameters with intra-plane phasing are used to simulate the temporal resolution of the data. As a result, this proxy dataset provides a practical set of data that very 


\section{HNR SAT: 00 Mon Aug 01 04:35:00 2005}

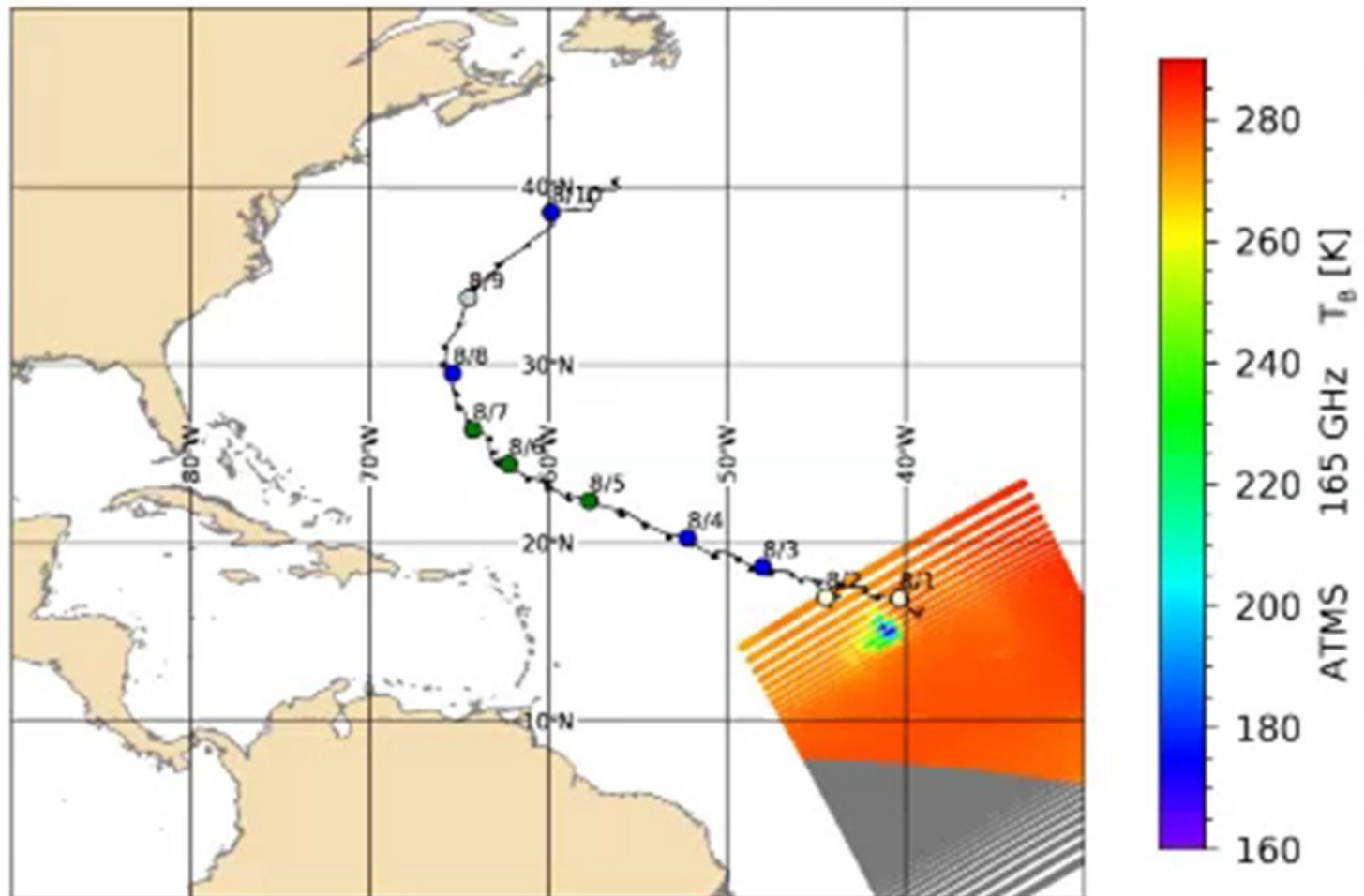

OTS/TD OCat 1 oCat 2 ocat 3 OCat 4 ocat 5

Figure 4. Sample TROPICS Proxy dataset demonstrating the spatial simulation of the data over the early stages of the simulated hurricane. Gray areas represent areas of the simulated swath that fall outside the domain of the hurricane nature run.
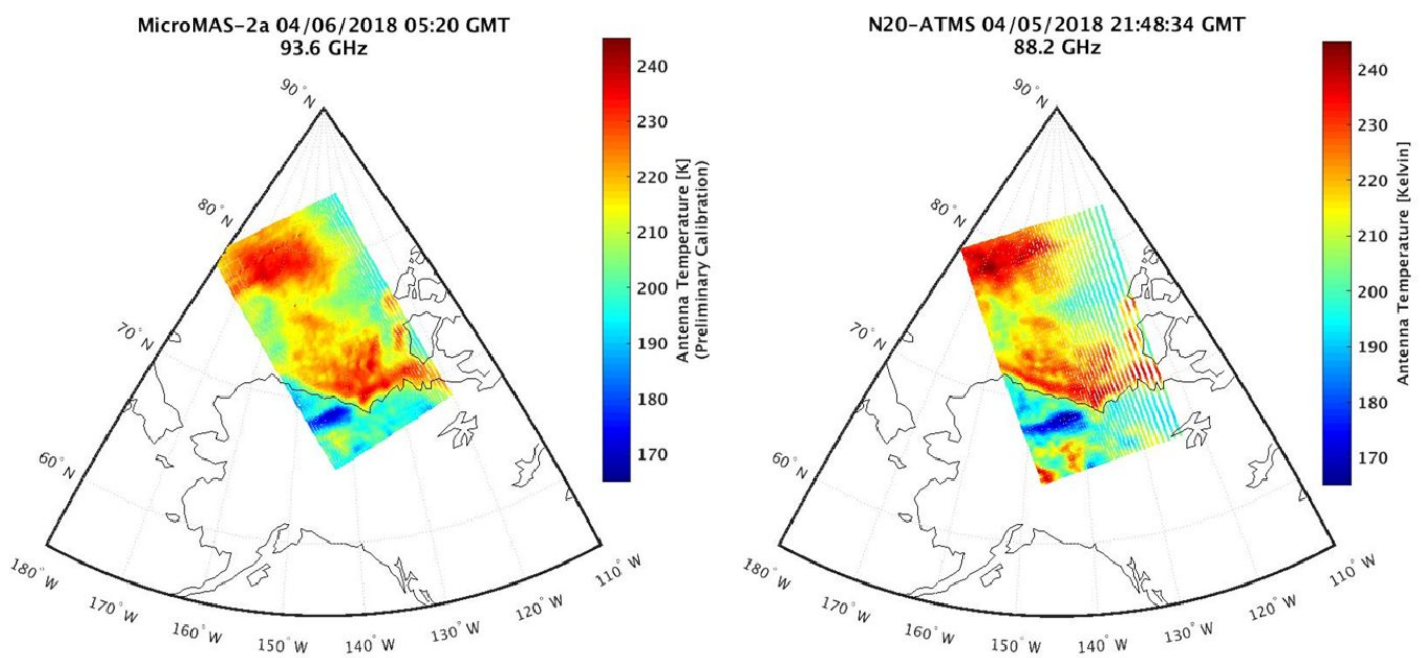

Figure 5. MicroMAS-2a on-orbit data (left) compared to NOAA-20 ATMS data (right) observed approximately seven hours earlier. 
closely replicates the spatial, temporal, and spectral resolution expected from TROPICS. Figure 4 shows a snapshot of a single swath of TROPICS proxy data derived from the HNR.

Beyond the HNR, data from MicroMAS-2a/b are also candidates for being used as proxy datasets for TROPICS. An initial analysis comparing MicroMAS-2a to the Advanced Technology Microwave Sounder (ATMS) ${ }^{12}$ over the same location is shown in Fig. 5 for the W-band. The ATMS data are masked in order to provide a better comparison to the MicroMAS-2a data because of the narrower swath MicroMAS-2a swath width. The location of the imagery is an ice sheet over Alaska that is breaking up into the ocean. The comparison between MicroMAS-2a data takes place over seven hours apart; thus, it provides a qualitative analysis but radiance validation needs to be completed in order to provide a quantitative analysis of the CubeSat data.

In addition to the science team investigation of the mission science objectives, an applications community for TROPICS has been developed. The types of applications and the community of users began with an applications workshop where users learned about the TROPICS mission and its data and were able to contribute potential user communities in their areas of application expertise ${ }^{13}$. Following this workshop, a first set of Early Adopters was established. An Early Adopter is defined as an unfunded applied scientist and/or decision maker recognized by the science team as a formal user of the data enabling early access to proxy data and to the science team. Through distribution of proxy data with a file structure and format similar to the expected mission data, it allows users to begin constructing data readers and procedures for how to ingest the data into models or other decision support tools to accelerate post-launch use.

\section{SUMMARY}

The NASA TROPICS mission uses heritage passive cross-track scanning microwave radiometers called MicroMAS-2 flown aboard a constellation of $3 \mathrm{U}$ CubeSats to enable rapid temporal sampling of the tropics with global coverage. This enhanced temporal resolution of PMW data is expected to help answer long-standing questions in the science community related to rapid intensification in TCs. TROPICS is currently on schedule for launch readiness in late 2019 with potential launch opportunities expected in 2020. Observations will be made in 12 channels from 90 to $205 \mathrm{GHz}$ that will enable a number of science questions to be addressed. Data will be made available to the public through NASA GES DAAC and proxy datasets are being developed to support early investigations into the ability of the data to address the science questions and potentially accelerate integration into decision support if latency issues can be overcome.

\section{REFERENCES}

[1] Schmidt, T. J., Gunshor, M. M., Menzel, W. P., Gurka, J. J., Li, J., and Bachmeier, S., "Introducing the nextgeneration Advanced Baseline Imager (ABI) on GOES-R.”, Bull. Amer. Meteor. Soc., 8, 1079-1096, (2005).

[2] Schmidt, T. J., Lindstrom, S. S., Gerth, J. J., Gunshor, M. M., "Applications of the 16 spectral bands on the Advanced Baseline Imager (ABI)", J. Operational Meteor., 6 (4), 33-46, (2018).

[3] Blackwell, W. J., et al., "An Overview of the TROPICS NASA Earth Venture Mission." Quarterly Journal of the Royal Meteorological Society, in press, (2018).

[4] Blackwell, W. J., "Technology Development for Small Satellite Microwave Atmospheric Remote Sensing," Proc. IEEE International Microwave Symposium, Honolulu (2017).

[5] Hou, A. Y., Kakar, R. K., Neeck, S., Azarbarzin, A. A., Kummerow, C. D., Kojima, M., Oki, R., Nakamura, K., and Iguchi, T., "The Global Precipitation Measurement Mission," Bull. Amer. Meteor. Soc. 95, 701-722 (2014).

[6] Desbois, J., Roca, R., Eymard, L., Vitard, N., Viollier, M., Srinivasan, J., and Narayanan, S., "The MeghaTropiques Mission," Proc. SPIE, 4899 (2003).

[7] Biswas, S. K., Farrar, S., Gopalan, K., Santos-Garcia, A., Jones, W. L., and Bilanow, S., "Intercalibration of Microwave Radiometer Brightness Temperatures for the Global Precipitation Measurement Mission," IEEE Trans. Geosci. Remote Sensing, 51(3), 1465-1477 (2013).

[8] Saunders, R. W., Blackmore, T. A., Candy, B., Francis, P. N., and Hewison, T. J., "Monitoring Satellite Radiance Biases Using NWP Models." IEEE Trans. Geosci. Rem. Sensing, 51(3), 1124-1138 (2013).

[9] Nolan, D. S., Atlas, R., Bhatia, K. T., and Bucci, L. R., "Development and validation of a hurricane nature run using the joint OSSE nature run and the WRF model," J. Adv. In Model. Earth Syst., 5, 382-405 (2013). 
[10] Skamarock, W. C., Klemp, J. B., Dudhia, J., Gill, D. O., Barker, D. M., Duda, M. G., Huang, X.-Y., Wang, W., and Powers, J. G., "A Description of the Advanced Research WRF Version 3," NCAR Tech Note. Note NCAR/ TN-475+STR, (2008).

[11] Han, Y., van Delst, F. P., Liu, Q., Weng, F., Yan, B., Treadon, R., and Derber, J. JCSDA Community Radiative Transfer Model (CRTM)-Version 1, NOAA Tech Rep. 122, NOAA, Silver Spring, MD, (2006).

[12] Goldberg, M. D., Kilcoyne, H., Cikanek, H., and Mehta, A., "Joint Polar Satellite System: The United States next generation civilian polar-obiting environmental satellite system", J. Geo. Res. Atmos., 118(13), 463-475, (2013)

[13] Zavodsky, B. T., et al., "First Time-Resolved Observations of Precipitation structure and storm Intensity with a Constellation of SmallSats (TROPICS) Mission Applications Workshop Summary Report," NASA Report NASA/CP-2017-219705, (2017). 